Elsevier Editorial System(tm) for Progresos de Obstetricia y Ginecología.

Manuscript Draft

Manuscript Number:

Title: Endometriosis umbilical

Article Type: Casos clínicos

Keywords: PALABRAS CLAVE: endometriosis, endometriosis umbilical, nódulo umbilical. KEYWORDS: endometriosis, umbilical endometriosis, umbilical node.

Corresponding Author: Dr. Manuel Angel Veiga Tuimil, Facultativo Especialista de AreaSanitari

Corresponding Author's Institution: Hospital A Marcide- Ferrol

First Author: Manuel Angel Veiga Tuimil, Facultativo Especialista de AreaSanitari

Order of Authors: Manuel Angel Veiga Tuimil, Facultativo Especialista de AreaSanitari; Verónica Miguez; Silvia Rodriguez; María Teresa Mosquera; María Calaza; Javier M-P-Mendaña

Abstract: RESUMEN: la endometriosis umbilical es la forma clínica más común de endometriosis extrapélvica y el tipo más habitual de endometriosis cutánea. Puede ser primaria ó secundaria. Las endometriosis umbilicales secundarias se producen sobre cicatrices de laparotomías o laparoscopias en las que ha habido exposición al tejido endometrial. La endometriosis umbilical es relativamente rara pero se debe tener en cuenta al realizar el diagnóstico diferencial de lesiones de localización umbilical. Presentamos un caso de endometriosis umbilical en una mujer de 39 años sin antecedentes de cirugía abdominal previa.

ABSTRACT: Umbilical endometriosis is the most common clinical form of extrapelvic and cutaneous endometriosis. It can be primary or secondary. Secondary umbilical endometriosis is produced in surgical scars of laparotomy or laparoscopy with exposure to endometrial tissue. Umbilical endometriosis is a rare disease but it must be considered in differential diagnosis of an umbilical node. We report a case of umbilical endometriosis in a 39 years old woman without previous abdominal surgery. 


\section{Carta de presentación}

Sr director:

Le envió un artículo para que considere su publicación en la sección de "casos clínicos" de la revista Progresos de Obstetricia y Ginecología. Describimos un caso clínico de endometriosis umbilical, una entidad poco frecuente, pero cuya prevalencia está aumentando en los últimos años debido al aumento de la cirugía abdominal con exposición al endometrio. Nuestra paciente sufría una endometriosis umbilical primaria, es decir no había intervenciones quirúrgicas previas. Consideramos que los ginecólogos deben conocer esta patología a pesar de su baja prevalencia porque la pueden encontrar cada vez con mayor frecuencia. 
Título: endometriosis umbilical.

Autores: Manuel Ángel Veiga, Verónica Míguez, Silvia Rodríguez, María Teresa Mosquera (*), María Calaza, Javier M-P-Mendaña.

Servicio de Ginecología y Obstetricia.

$\left({ }^{*}\right)$ Servicio de Anatomía Patológica.

Hospital Arquitecto Marcide.

Área Sanitaria de Ferrol.

C/ Carretera de Catabois sn 15405 Ferrol

Correspondencia:

Manuel Angel Veiga Tuimil.

Teléfono: 626552626.

Correo electrónico: manuel.angel.veiga.tuimil@sergas.es.

Dirección: C/Paraguay 10-12, 3애 15404 Ferrol. 
ENDOMETRIOSIS UMBILICAL.

RESUMEN: la endometriosis umbilical es la forma clínica más común de endometriosis extrapélvica y el tipo más habitual de endometriosis cutánea. Puede ser primaria ó secundaria. Las endometriosis umbilicales secundarias se producen sobre cicatrices de laparotomías o laparoscopias en las que ha habido exposición al tejido endometrial. La endometriosis umbilical es relativamente rara pero se debe tener en cuenta al realizar el diagnóstico diferencial de lesiones de localización umbilical. Presentamos un caso de endometriosis umbilical en una mujer de 39 años sin antecedentes de cirugía abdominal previa.

ABSTRACT: Umbilical endometriosis is the most common clinical form of extrapelvic and cutaneous endometriosis. It can be primary or secondary. Secondary umbilical endometriosis is produced in surgical scars of laparotomy or laparoscopy with exposure to endometrial tissue. Umbilical endometriosis is a rare disease but it must be considered in differential diagnosis of an umbilical node. We report a case of umbilical endometriosis in a 39 years old woman without previous abdominal surgery.

PALABRAS CLAVE: endometriosis, endometriosis umbilical, nódulo umbilical.

KEYWORDS: endometriosis, umbilical endometriosis, umbilical node. 
INTRODUCCIÓN:

La endometriosis es la presencia de glándulas y/o estroma endometriales fuera de la cavidad endometrial. Fue descrita por Rokitansky en 1860. Se estima que afecta al 5-10\% de las mujeres en edad reproductiva $(1,2)$. La mayoría de las veces se produce en órganos pélvicos y puede ser asintomática o causar dolor pélvico crónico, dispareunia, dismenorrea y esterilidad. Se han descrito lesiones de endometriosis en casi todos los órganos y tejidos del cuerpo humano incluidos el cerebro y los pulmones.

La endometriosis umbilical (EU) es una forma de endometriosis extragenital descrita por Villar en 1886. Su incidencia se estima entre el 0,5 y 1,2 \% de todas las pacientes con endometriosis (3) y representa el $20 \%$ de las endometriosis extragenitales (3). Puede ser espontánea, también Ilamada primaria, ó secundaria. La EU secundaria se produce en cicatrices quirúrgicas, sobre todo de laparotomías (cesáreas, histerectomías,...) o de laparoscopias, en las que ha habido exposición a tejido endometrial $(4,5,6,7)$. La EU secundaria es relativamente rara y la EU primaria es todavía menos frecuente (8).

La EU es la forma clínica más habitual de endometriosis extrapélvica (3) y el tipo más frecuente de endometriosis cutánea (9).

Presentamos un caso de EU primaria.

\section{DESCRIPCIÓN DEL CASO:}

Mujer de 39 años de edad, diagnosticada de síndrome del ovario poliquístico (criterios ESHRE 2003) sin antecedentes quirúrgicos. Tuvo un embarazo y parto normal a los 28 años. Refiere que desde hace 9 meses presenta dolor y sangrado en el ombligo coincidiendo con la menstruación. En la exploración se observa un nódulo de 5 milímetros de coloración azulada, doloroso a la palpación, que está sangrando (figura 1). El médico de atención primaria lo había criocoagulado sin mejoría. Ante el diagnóstico de sospecha de endometriosis umbilical se realizó extirpación quirúrgica del nódulo con anestesia local. El estudio anatomopatológico de la pieza quirúrgica confirmó el diagnóstico de endometriosis. En las figuras 2 y 3 se aprecian células y glándulas endometriales en el seno de un tejido dérmico.

No se encontró evidencia clínica o ecográfica de endometriosis en la pelvis o en otras localizaciones. La paciente pidió un método anticonceptivo seguro y se le prescribieron anovulatorios orales (etinilestradiol $20 \mathrm{mcg}$ / drosperinona $3 \mathrm{mg}, 24$ días por ciclo). Tras 4 años de seguimiento no volvieron a aparecer lesiones umbilicales ni hubo evidencia clínica o ecográfica de endometriosis en otras localizaciones.

DISCUSIÓN: 
La EU es un trastorno poco frecuente. Hasta 2007 se habían publicado en las revistas 2007 (3). En un subgrupo de 68 de estas pacientes había datos suficientes en las publicaciones para poder analizar la clínica. La forma de presentación de la EU fue muy variada, incluso 2 pacientes estaban asintomáticas. 90,9\% presentaban un nódulo umbilical quístico, de tamaño y coloración muy variable. El color de la lesión podía ser, por orden de frecuencia, marrón, azul, púrpura, rojo, negro o normal, y la piel circundante frecuentemente estaba eritematosa. $81,5 \%$ presentaban dolor, habitualmente cíclico, que empeoraba progresivamente; 49,2\% presentaban sangrado umbilical, que coincidía casi siempre con la menstruación. La mayoría de las pacientes que tenían dolor también sangraban. Para Victori y cols. la EU se presenta característicamente como un nódulo umbilical de tamaño y coloración variable asociado a dolor y sangrado umbilical, que suele tener relación con la menstruación. Consideran esta forma de presentación patognomónica (3). En nuestro caso, aunque el nódulo era muy pequeño el modo de presentación fue típico.

Aunque el ombligo es el lugar más frecuente de endometriosis extrapélvica espontánea, la EU es poco común. En la primera mitad del siglo XX la mayoría de los casos publicados eran EU primarias (10). Actualmente, debido al gran aumento de los procedimientos quirúrgicos abdominales, es mucho más frecuente la EU secundaria $(4,5,6,7)$. Nuestra paciente nunca había sido intervenida quirúrgicamente.

La etiología y la patogenia de la endometriosis permanecen inciertas (2). Todavía no existe acuerdo acerca del origen celular o molecular de la enfermedad (11).La mayoría de los casos pélvicos se podrían explicar por la teoría de la menstruación retrógrada, planteada por Sampson en 1927 (12). Otras teorías como la de la diseminación hematógena o linfática, la de la metaplasia celómica, el origen a partir de células pluripotenciales de la médula ósea o la diseminación iatrogénica durante procedimientos quirúrgicos con exposición al endometrio explicarían el resto de los casos (13). Cuando existe endometriosis pélvica concomitante la EU primaria se podría explicar por diseminación linfática o vascular (4). En el resto de los casos, la mayoría, se ha sugerido que podría ser debida a metaplasia de restos embrionarios del uraco (14). La endometriosis secundaria se debe a implantes endometriales en cicatrices de laparotomías o de laparoscopias en pacientes sometidas a cirugía con exposición al endometrio $(4,5,6,7)$.

El riesgo de degeneración maligna de las lesiones endometriosicas umbilicales es extremadamente bajo $(3,14)$.

Aunque se han utilizado múltiples métodos de imagen (ecografía, tomografía computada, resonancia magnética) ninguno es específico. El diagnóstico definitivo de la endometriosis es anatomopatológico (15). Aunque algunos autores realizan punción aspiración con aguja fina la sensibilidad es baja y los resultados habitualmente no son concluyentes $(4,16)$.

El tratamiento de elección es la extirpación de la lesión $(3,14)$. Tras la cirugía la tasa de recurrencias es muy baja. Aunque hay muy pocos casos publicados el tratamiento médico con progesterona, danazol, gestrinona o noretisterona, en general, no ha sido eficaz. El uso de análogos de la GnRH preoperatorios se asocia a resección incompleta de la lesión (17). 
Solo el $15 \%$ de las EU primarias se asocian a endometriosis pélvica confirmada (3). Debido a esta baja tasa de endometriosis pélvica simultánea la laparoscopia diagnóstica solo está indicada en pacientes con síntomas o signos de enfermedad genital.

El diagnóstico diferencial se debe hacer con hernia umbilical, quiste del uraco, granulomas piógenos o por cuerpo extraño, nevus dérmico, malformaciones congénitas del conducto onfalomesentérico, carcinoma umbilical metastásico o primario, melanoma umbilical, eventración, hematoma, absceso...

Como conclusión se debe decir que la EU primaria es una entidad poco frecuente pero que se debe tener en cuenta al realizar el diagnóstico diferencial de lesiones localizadas en el umbiligo. 
BIBLIOGRAFIA:

1-Waller KG, Lindsay P, Curtis P, Shaw RW. The prevalence of endometriosis in women with infertile partners. Eur J Obstet GynecolReprod Biol 1993;48:135-9.

2-Giudice LC, Kao LC. Endometriosis. Lancet 2004; 364:1789-99.

3-Victori R, Diamond MP, Johns A. Villar's nodule: a case report an systematic literature review of endometriosis externa of the umbilicus. J Minim Invasive Gynecol 2007;14:23-32.

4-De Giorgi V, Massi D, Mannone F, Stante M, Carli P. Cutaneous endometriosis: non-invasive analysis by epiluminescence microscopy. Clin Exp Derm 2003;28:315-17.

5-Teh W-T, Vollenhoven B, Harris PI. Umbilical endometriosis, a pathology that gynecologists may encounter when inserting the Veres needle. Fertil Steril 2006;86:1764e1-2.

6-Koninckx PR, donders G, Vandecruys $\mathrm{H}$. Umbilical endometriosis after unprotected removal of uterine pieces throught the umbiligus. J Am Assoc Gynecol Laparosc 2000;7:227-32

7- González-Santín V, Robres J, Farreras N, Ortiz de Zárate L, Buqueras C, Bachs E y Flores J. Endometriomas de la pared abdominal. Revisión de una serie de 17 casos Clin Invest Gin Obst 2008;35:2-6.

8-Hidalgo LA, Heredia A, Fantova MJ, Badia JM y Suñol X. Endometriomas de pared abdominal: aspectos diagnósticos. Cir Esp 2001; 70: 34-7.

9-Albrecht LE, Tron V, Rivers JK. Cutaneous endometriosis. Int J Dermatol 1995;34:261-2.

10-Steck WD, Helwig EB. Cutaneous endometriosis. JAMA 1965; 191:167-70.

11-Bulum SE. Endometriosis. N Engl J Med 2009;360:268-79.

12- Sampson JA. Peritoneal endometriosis due to menstrual dissemination of endometrial tissue into the peritoneal cavity. Am J Obstet Gynecol 1927;14:422-69.

13- Sasson IE, Taylor HS. Stem cells and the pathogenesis of endometriosis. Ann N Y Acad Sci 2008;1127:106-15.

14-Latcher JW. Endometriosis of the umbilicus. Am J Obstet Gynecol 1953; 66:161-8.

15 - ACOG. Management of endometriosis. ACOG Practice Bulletin no 114. Obstet Gynecol 2010; 114:223-35.

16-Zhao X, Lang J, Leng J, Liu Z, Sun D y Zhu L. Abdominal wall endometriomas. Int J Gynaecol Obstet 2005; 90:218-22.

17- Agarwal A, Fong YF. Cutaneous endometriosis. Singapore Med J 49:704-9. 
PIÉS DE FIGURA.

Figúra1: nódulo umbilical endometriósico.

Figúra 2: tinción con hematoxilina. Glándulas y células endometriales en el seno de un tejido dérmico.

Figúra 3: tinción para receptores de estrógenos. Glándulas y células endometriales en el seno de un tejido dérmico. 
Figura 1
Click here to download high resolution image

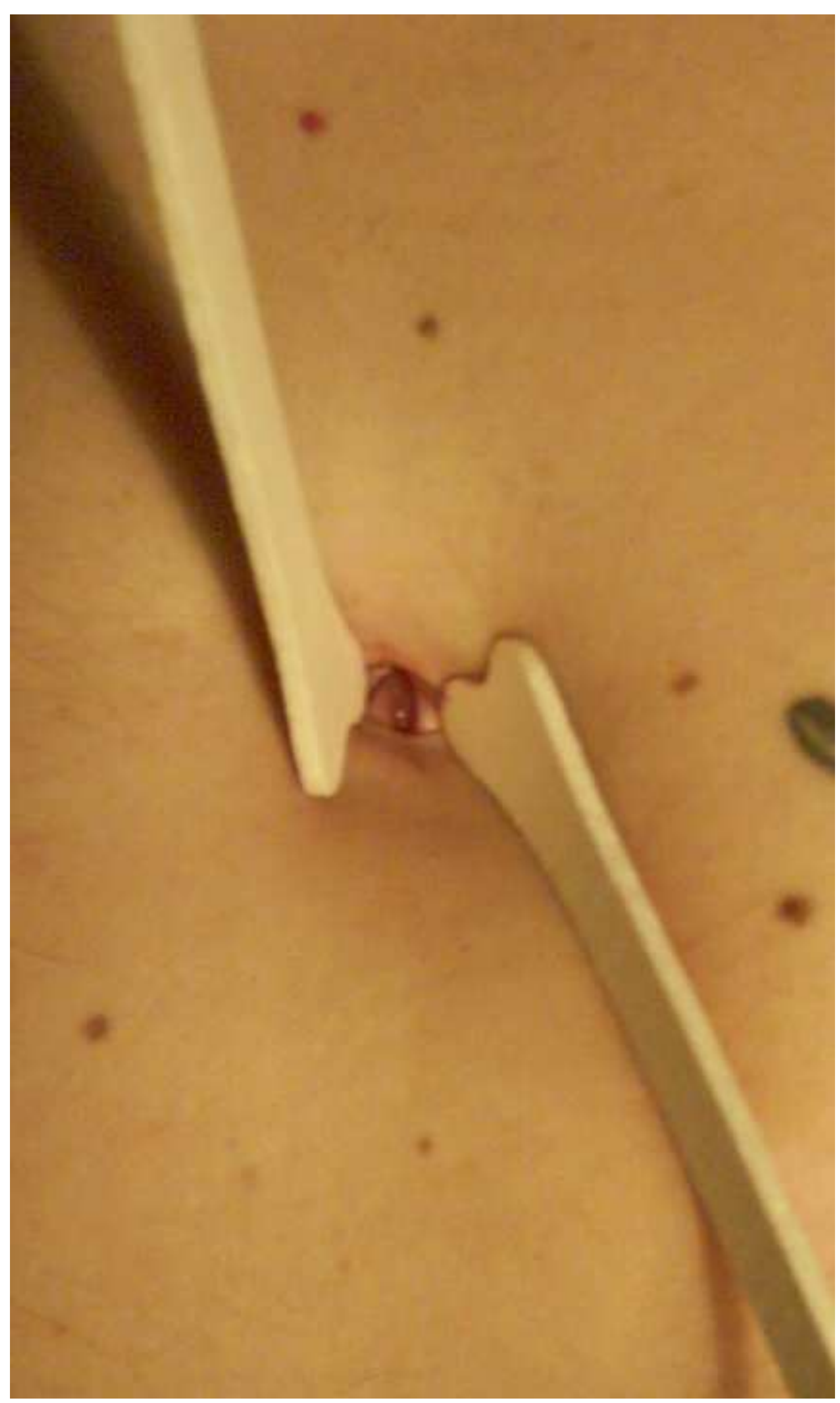



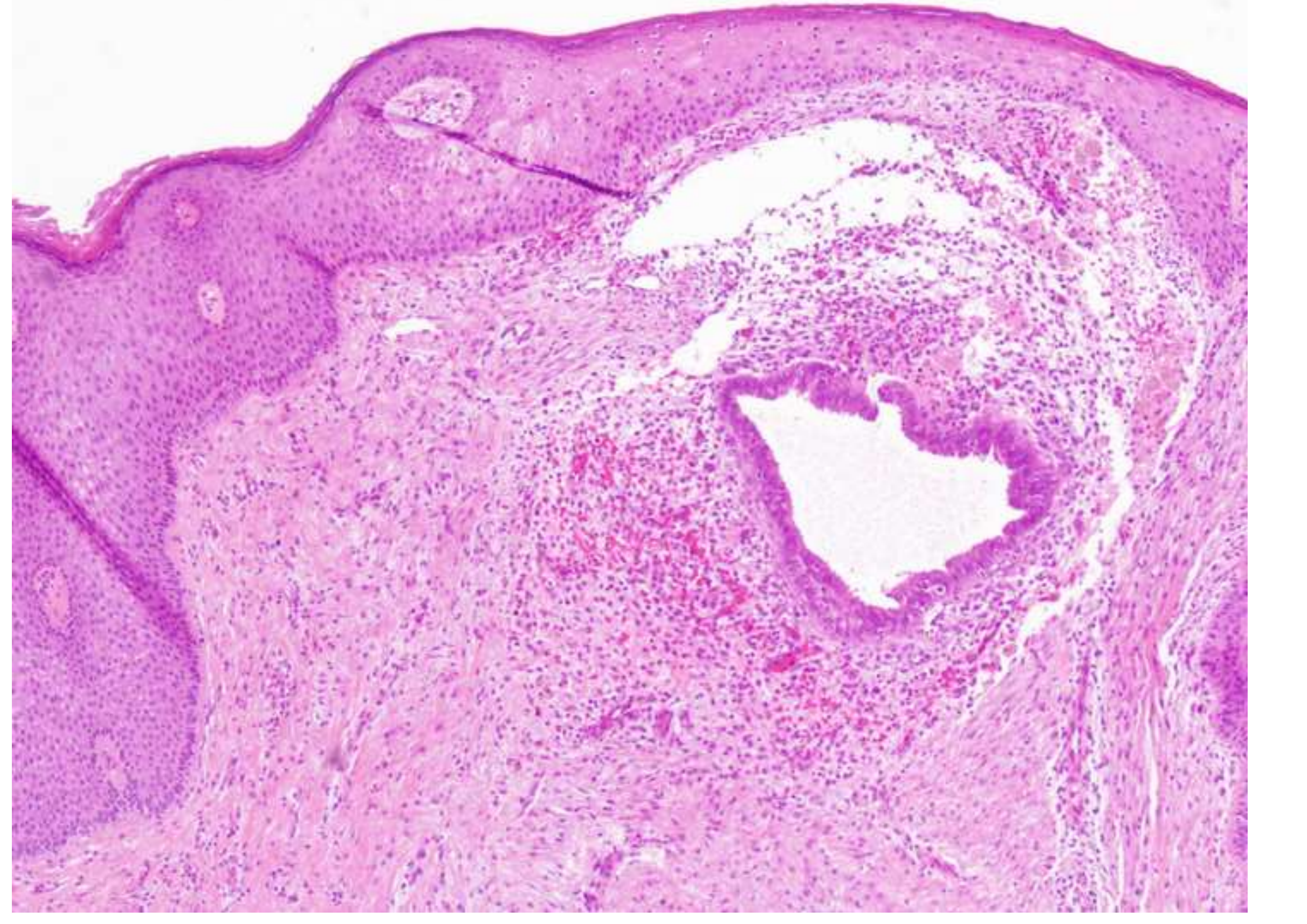

.

.

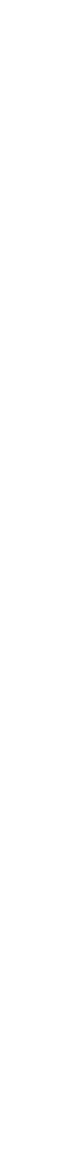


